\title{
Effect of Nano-Ni Catalyst on the Growth and Characterization of Diamond Films by HFCVD
}

\author{
Chien-Chung Teng, ${ }^{1}$ Feng-Chi Ku, ${ }^{2}$ Chien-Min Sung, ${ }^{2}$ Jin-Pei Deng, ${ }^{3}$ Su-Fang Chien, ${ }^{3}$ \\ Shin-Min Song, ${ }^{4}$ and Chhiu-Tsu Lin ${ }^{1}$ \\ ${ }^{1}$ Department of Chemistry and Biochemistry, Northern Illinois University, Dekalb, IL 60115, USA \\ ${ }^{2}$ Chemical Vapor Deposition Division, Research and Development Department, Kinik Company, No. 64, Chung-San Road, \\ Ying-Ko, Taipei 239, Taiwan \\ ${ }^{3}$ Department of Chemistry, Tamkung University, Tamsui, Taipei 239, Taiwan \\ ${ }^{4}$ Department of Mechanical Engineering, Northern Illinois University, Dekalb, IL 60115, USA
}

Correspondence should be addressed to Chhiu-Tsu Lin, ctlin@niu.edu

Received 23 July 2009; Accepted 4 March 2010

Academic Editor: Rakesh Joshi

Copyright ( $\odot 2010$ Chien-Chung Teng et al. This is an open access article distributed under the Creative Commons Attribution License, which permits unrestricted use, distribution, and reproduction in any medium, provided the original work is properly cited.

\begin{abstract}
Four different catalysts, nanodiamond seed, nano-Ni, diamond powder, and mixture of nano-Ni/diamond powder, were used to activate Si wafers for diamond film growth by hot-filament CVD (HFCVD). Diamond crystals were shown to grow directly on both large diamond powder and small nanodiamond seed, but a better crystallinity of diamond film was observed on the ultrasonicated nanodiamond seeded Si substrate. On the other hand, nano-Ni nanocatalysts seem to promote the formation of amorphous carbon but suppress transpolyacetylene (t-PA) phases at the initial growth of diamond films. The subsequent nucleation and growth of diamond crystals on the amorphous carbon layer leads to generation of the spherical diamond particles and clusters prior to coalescence into continuous diamond films based on the $\mathrm{CH} 3$ addition mechanism as characterized by XRD, Raman, ATR/FT-IR, XPS, TEM, SEM, and AFM techniques. Moreover, a 36\% reduction in surface roughness of diamond film assisted by nano-Ni catalyst is quite significant.
\end{abstract}

\section{Introduction}

Recently, Ni nanoparticles were utilized for catalytic synthesis of carbon nanotube (CNT) by CVD because the graphitic layer covering $\mathrm{Ni}$ nanoparticles would transform into CNT under a controlled temperature $[1,2]$. On the other hand, the catalytic effect of $\mathrm{Ni}$ also was illustrated to inhibit CVD diamond nucleation and growth, though diamond may nucleate and grow on the amorphous carbon or graphitic interlayer initially formed on $\mathrm{Ni}$ in a low-pressure methanehydrogen environment [3-7]. Consequently, under a wellcontrolled growing condition, $\mathrm{CNT}$ and diamond can be simultaneously and selectively grown on $\mathrm{Ni}$-coated and diamond powder-seeded Si substrates [8].

However, diamond powders and/or mixed with transition metal powders of size in micron scale (Ni of $3 \sim 5 \mu \mathrm{m}$ in size, $\mathrm{Ti}, \mathrm{Cu}, \mathrm{Fe}$, etc.) have also been processed as catalysts to enhance the initial nucleation density for diamond film growth by ultrasonication seeding and HFCVD $[9,10]$. The catalytic enhancement was explained to result from the dissociative chemisorption of $\mathrm{CH}_{4}$ at the Ni catalytic sites. Since the catalytic effect of Ni nanoparticles is expected to improve substantially due to higher surface to volume ratio as compared with $\mathrm{Ni}$ particles in micron size, the $\mathrm{Ni}$ nanoparticles would be an ideal catalytic seeds for the growth of nanocrystalline diamond (NCD) film by HFCVD due to the enhanced nucleation density with the assistance of $\mathrm{Ni}$ nanoparticles. The as-grown NCD films are expected to have a better surface roughness ( $\mathrm{Ra}$ ) as compared to microcrystalline diamond films due to smaller crystallites. The NCD films with better Ra would have many potential applications such as cutting tools.

In this paper, we employed four different catalysts, ultrasonicated nanodiamond seed $(4 \sim 50 \mathrm{~nm})$, nano-Ni, 
diamond powder $(250 \sim 350 \mathrm{~nm})$, and mixture of nano$\mathrm{Ni}$ /diamond powder, to treat $\mathrm{Si}$ wafers for investigating the effects of those nanocatalysts on diamond film growth by HFCVD method. A plausible growth mechanism is suggested to explain the catalytic effect of Ni nanoparticles on the initial growth stage of CVD diamond film by interpreting XRD, Raman, ATR/FT-IR, XPS, TEM, SEM, and AFM results.

\section{Experimental}

\subsection{Preparation of Nanocatalysts}

2.1.1. Nano-Ni Catalysts. Nano-Ni catalysts were prepared by a chemical reduction method and stabilized in ethylene glycol (EG) solutions containing poly(vinylpyrrolidone) (PVP) polymer. First, a $10 \mathrm{~mL}$ of $10 \mathrm{mM} \mathrm{NaH} \mathrm{PO}_{2} \cdot \mathrm{H}_{2} \mathrm{O}$ (J. T. Baker 3740) in an EG solution and a $10 \mathrm{~mL}$ of $4 \mathrm{mM}$ $\mathrm{NiSO}_{4} \cdot 6 \mathrm{H}_{2} \mathrm{O}$ (Fisher N73-100) in an EG solution were separately and freshly prepared and then well mixed by a magnetic stirrer for 30 minutes. Second, $0.37 \mathrm{wt} \%$ of PVP (SIGMA-ALDRICH 437190-500G) powder was added into the $\mathrm{NaH}_{2} \mathrm{PO}_{2} \cdot \mathrm{H}_{2} \mathrm{O} / \mathrm{NiSO}_{4} \cdot 6 \mathrm{H}_{2} \mathrm{O} / \mathrm{EG}$ solution and mixed for at least 1.5 hour. Third, the final solution was heated under microwave $(2.45 \mathrm{GHz}, 900 \mathrm{~W})$ for 3 minutes until the color of the solution changed from light green to black brown, indicating the formation of nano-Ni particles in solutions.

2.1.2. Diamond Powder Solution. First, $0.37 \mathrm{wt} \%$ of PVP was added into a $46 \mathrm{~g}$ EG solution and mixed by a magnetic stirrer for at least 1.5 hours. Second, $1 \mathrm{wt} \%$ of fluorosurfactant (FS, Dupont FS-510) was added into the PVP/EG solution and mixed for 30 minutes. Third, $0.005 \mathrm{wt} \%$ of nanodiamond seed (ND, Carbo-Tec Dynaget M3D) or diamond powder (NDP, SP-DP Maple Canada Group) was added into the FS/PVP/EG solution and well dispersed by a sonication dismembrator (Fisher Scientific Model-100) with $15 \mathrm{~W}$ power for 30 seconds.

2.2. Deposition of Diamond Films by HFCVD. The polished (100) Si substrates were pretreated with (a) nanodiamond seeds, ND $(4 \sim 50 \mathrm{~nm})$ by ultrasonication, (b) diamond powders, NDP $(250 \sim 350 \mathrm{~nm})$, (c) nano-Ni nanocatalysts, and (d) a mixture of nano-Ni and NDP. The surface modification processes (b), (c), and (d) were done by spin-coating (Photoresist spinner EC101D-R485, Headway Res. Inc.) using as-prepared nano-Ni/EG and diamond powder/EG solutions. The modified Si substrates were used for diamond films deposition by HFCVD system (Sp3 Inc., Model 500). The deposition pressure was controlled at 18 20 Torr, containing a gas mixture of $2.3 \mathrm{vol} \%$ methane (99.995\% purity) and hydrogen $(99.95 \%$ purity) under a total flow rate of $3000 \mathrm{sccm}$. The distance between the tungsten (W) filament and $\mathrm{Si}$ substrates was adjusted at $10 \mathrm{~mm}$. The W filament temperature was controlled at 2000 $\sim 2200^{\circ} \mathrm{C}$. The temperature of $\mathrm{Si}$ substrates was about 650 $\sim 700^{\circ} \mathrm{C}$ in the seeding stage and about $800 \sim 850^{\circ} \mathrm{C}$ in the growing stage. In this work, an initial nucleation time of 1.5 hours and a total deposition time of 48 hours were employed.

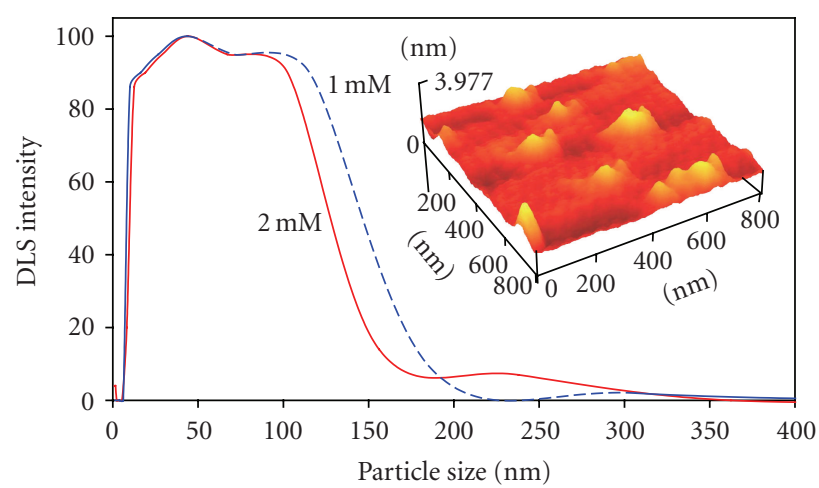

FIgure 1: Particle size distribution by DLS and AFM of nano-Ni catalysts.

2.3. Characterization Methods. A dynamic light scattering (DLS) goniometer equipped with a HeNe laser at $630 \mathrm{~nm}$ (Brookhaven Instruments, Model BI-200SM) was used to characterize the size distribution of nano-Ni particles in EG solutions. The lattice structure of diamond films was analyzed by X-ray powder diffraction instrument (XRD, Rigaku Corp., Model MiniFlex). Both the exposed surface of diamond film (front-side) and the interface between diamond film and Si substrate (backside) were examined. Renishaw Raman Scattering Noodles System 2000, equipped with a Leica microscope and an $\mathrm{HeNe}$ red $(632.8 \mathrm{~nm})$ as an excitation laser source, was used to examine the molecular properties and quality of diamond films from both the front and backsides. ATR/FT-IR spectrometer (PIKE, MIRacle Single Reflection Horizontal ATR Accessory equipped with $1.8 \mathrm{~mm}$ round $\mathrm{ZnSe}$ crystal IRE plate) was used to diagnose the molecular vibrations of diamond films in mid-IR $\left(650 \sim 4000 \mathrm{~cm}^{-1}\right)$ range recorded with 100 scans and $4 \mathrm{~cm}^{-1}$ resolution. X-ray photoelectron spectroscopy $\left(\mathrm{Mg}-\mathrm{K}_{\alpha}\right.$ radiation of 1253.6) was used to characterize the binding environments of carbon 1s core level in diamond films without $\mathrm{Ar}^{+}$sputtering surface pretreatment. Planview Transmission electron microscopy (TEM, JEOL, JSM$1200 \mathrm{EX}$ II) pictures were taken to identify the possible growing structures and/or phases of diamond films. Scanning electron microscopy (SEM, JEOL JSM-5600) micrographs were taken to illustrate the surface morphology of diamond films. Atomic force microscopy (AFM, Quesant Q-scope 350) images were scanned for the surface roughness of diamond films.

\section{Results and Discussions}

3.1. Size Distribution of Nano-Ni Catalysts with PVP in EG by DLS and AFM. DLS results indicated a mean diameter of around $50 \mathrm{~nm}$ (Figure 1) for both $1 \mathrm{mM}$ (dash blue) and $2 \mathrm{mM}$ (solid red) of nano-Ni catalysts with $0.37 \mathrm{wt} \% \mathrm{PVP}$ in EG solutions. In the insert of Figure 1, the AFM morphology of the nano-Ni catalysts spun-coated on $\mathrm{Si}$ substrate also showed the particle size around $50 \sim 70 \mathrm{~nm}$. Those nano$\mathrm{Ni}$ catalysts were used to assist diamond film growth on $\mathrm{Si}$ by HFCVD. 


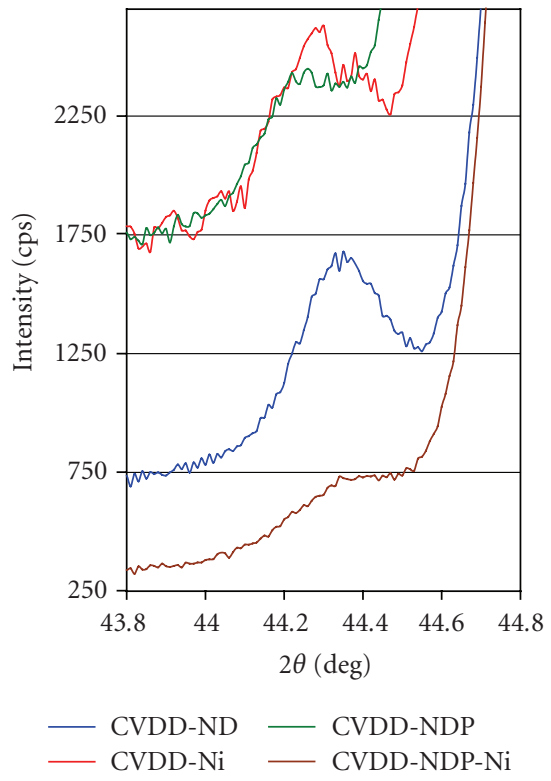

(a)

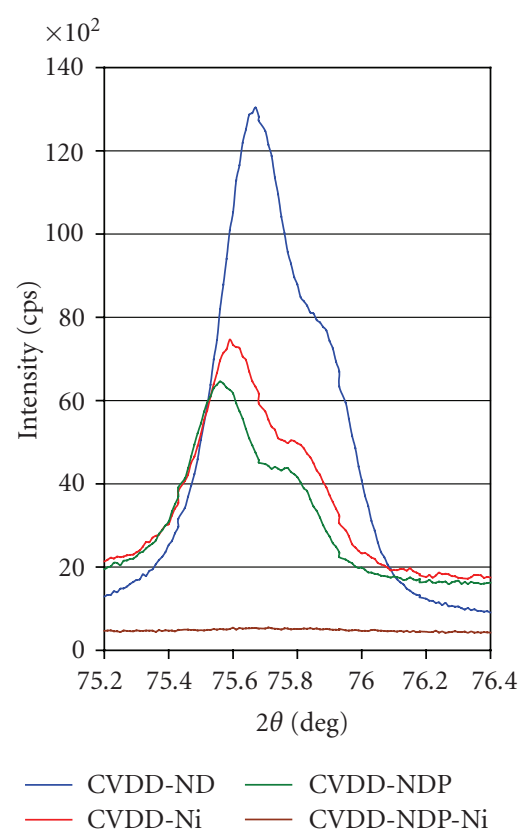

(b)

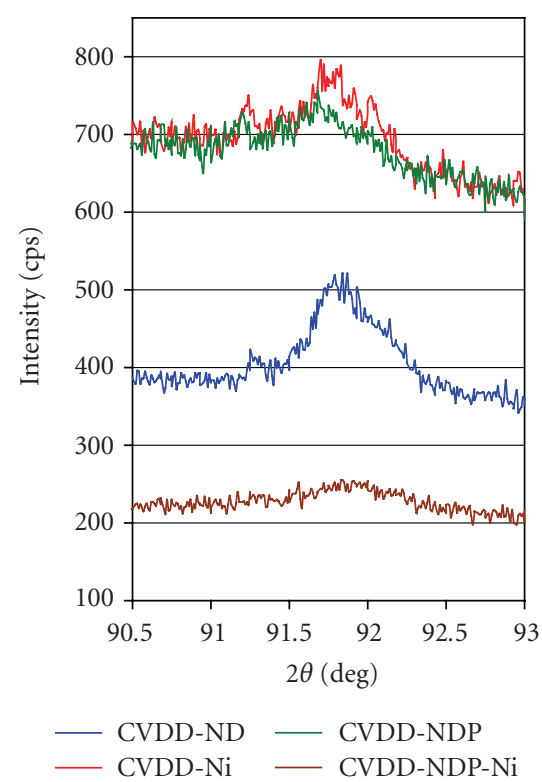

(c)

FIGURE 2: XRD of diamond films: (a) $\langle 111\rangle$, (b) $\langle 220\rangle$, and (c) $\langle 311\rangle$ planes.

3.2. XRD of Diamond Films. Figure 2 shows XRD patterns of the front-sides of diamond films (catalyzed by ND: blue; nano-Ni: redl; NDP: green; and a mixture of nano$\mathrm{Ni} / \mathrm{NDP}$ - brown), which are labeled as CVDD-ND, CVDD$\mathrm{Ni}$, CVDD-NDP, and CVDD-NDP-Ni, respectively. The XRD spectra (Figures 2(a), 2(b), and 2(c)) display three characteristic peaks around $44.3^{\circ}, 75.6^{\circ}$, and $91.8^{\circ}$ in $2 \theta$, which are assigned to $\langle 111\rangle,\langle 220\rangle$, and $\langle 311\rangle$ lattice planes of diamond crystals. The (220) reflection being considerably more intense than (111) reflection suggests the formation of pyramidal faceted diamond crystals as observed by SEM in Figure 8. The XRD, for the backsides of diamond films are not presented here. Both $\langle 111\rangle$ and $\langle 311\rangle$ planes are not observed. However, a small bump around $75.5^{\circ}(\langle 220\rangle$ plane) in $2 \theta$ of diamond crystal seems to come out at the initial growth stage. This observation may suggest the phase transition from the nondiamond to diamond structures during the growth of diamond films. As shown in Figure 2, the crystallinity of CVDD-ND diamond films is better than the others. However, the crystallinity of CVDDNDP-Ni diamond film is extremely weak. This may come from Ni catalytic graphitization of diamond powders, which facilitates the formation of amorphous phase in the early growth stage of diamond film, as shown to give an almost featureless XRD in Figure 2.

All XRD peaks observed in Figure 2 are quite broad (FWHM $0.3^{\circ} \sim 0.5^{\circ}$ in $2 \theta$ ), suggesting the formation of small diamond crystals. The observed XRD peaks displayed peak shifts of $2 \theta$ about $0.3^{\circ} \sim 0.4^{\circ}$ relative to the corresponding theoretical positions that may imply the inhomogeneous stresses existed in diamond films. In Figure 2(b), the peak shoulders around $75.8^{\circ}$ in $2 \theta$ can possibly result from the stacking faults. As in Figure 2(a), a shoulder around $44.3^{\circ}$ in $2 \theta$ for $\langle 111\rangle$ peak for nano-Ni-assisted diamond films (CVDD-Ni) may be due to the lattice mismatching between diamond crystals and nano-Ni catalysts.

3.3. Raman Spectra of Diamond Films. Molecular properties of both front and backsides of diamond films (CVDD-ND: blue; CVDD-Ni: red; CVDD-NDP: green) were examined by Raman scattering as shown in Figure 3, where the backside gave the chemical characteristics of the diamond film at the initial growth stage. An amorphous carbon phase starts to form at the initial stage as observed by a small broad band at $1520 \mathrm{~cm}^{-1}$ on the backside (the insert in Figure 3(b)). This is then transformed to give the diamond structure as displayed by a small peak around $1334 \mathrm{~cm}^{-1}$ on the front side (the insert in Figure 3(a)). In all cases, the crystalline diamond peak at $1334 \mathrm{~cm}^{-1}$ is only weakly observed, suggesting the $\%$ and size of diamond in film composition is low and small. From Figure 3(a), Raman spectra of front-sides of diamond films indicate a better crystalline diamond feature in CVDD-ND than the others as evidenced by XRD in Figure 2. Contrarily, the backside of CVDD-Ni diamond film shows the most amorphous feature at the early growth.

All spectra showed an intense band at $2270 \mathrm{~cm}^{-1}$ and a shoulder band at $2800 \mathrm{~cm}^{-1}$, which have been attributed to several origins: (1) a photoluminescence peak or a color center due to silicon defects in diamond films [11, 12], and (2) the combinational overtone Raman band of a short conjugation chain of transpolyacetylene (t-PA), which may grow around the grain boundaries or surface of diamond films [13-17]. The origin (2) is preferred since these Raman bands have also been observed for the diamond films grown on nonsilicon substrates [18]. Moreover, in Figure 3, the intensity of $2270 \mathrm{~cm}^{-1}$ peak is even stronger on the front 


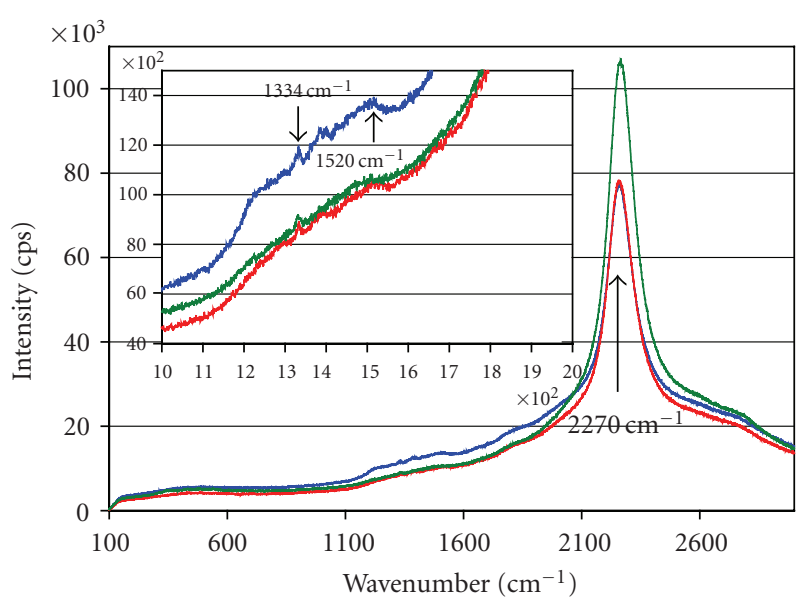

(a)

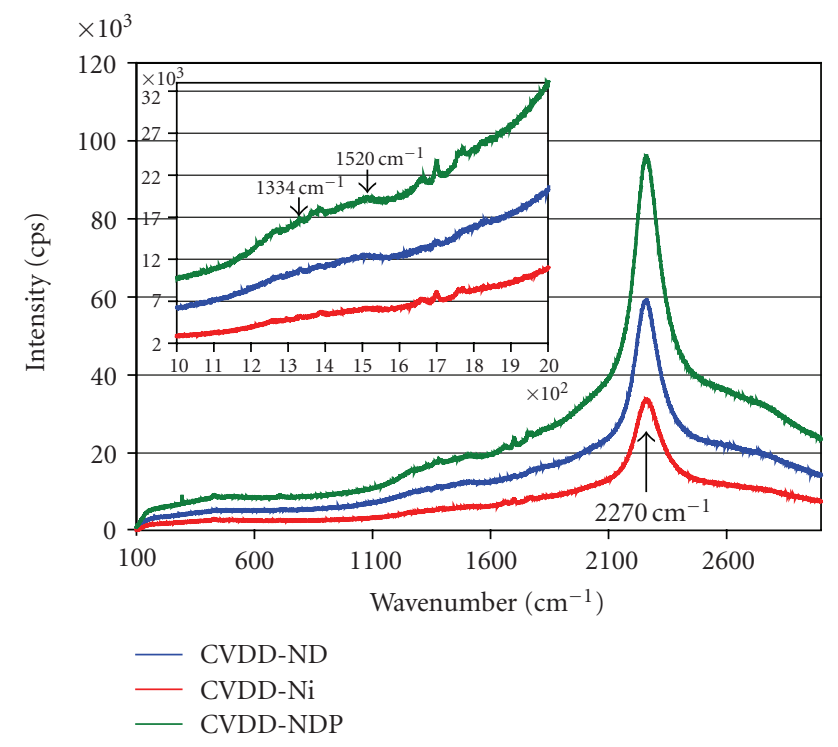

(b)

FIGURE 3: Raman spectra of front surfaces (a) and backsides (b) of diamond films.

than on the backsides of diamond films grown on Si by HFCVD. This indicates that the $2270 \mathrm{~cm}^{-1}$ peak is not due to the Si substrate defects, but the t-PA formed around the grain boundaries or surface of diamonds. It is worthwhile to mention that the backside of diamond film catalyzed by nano-Ni (CVDD-Ni, red) gives the lowest peak intensity of Raman band at $2270 \mathrm{~cm}^{-1}$, indicating that the formation of t-PA is low at the initial growth of diamond phase due to nano-Ni catalysts. This may come from the catalytic hydrogenation that converts t-PA into methylene-like chains in the presence of nano-Ni catalysts.

3.4. ATR/FT-IR Spectra of Diamond Films. Figure 4 shows ATR/FT-IR spectra of CVDD-ND (blue), CVDD-Ni (red), CVDD-NDP (green), and CVDD-NDP-Ni (brown) diamond films in the mid-IR range of $650 \sim 4000 \mathrm{~cm}^{-1}$. The absorption of water $\left(3500 \sim 3900 \mathrm{~cm}^{-1}\right.$ and $1400 \sim$

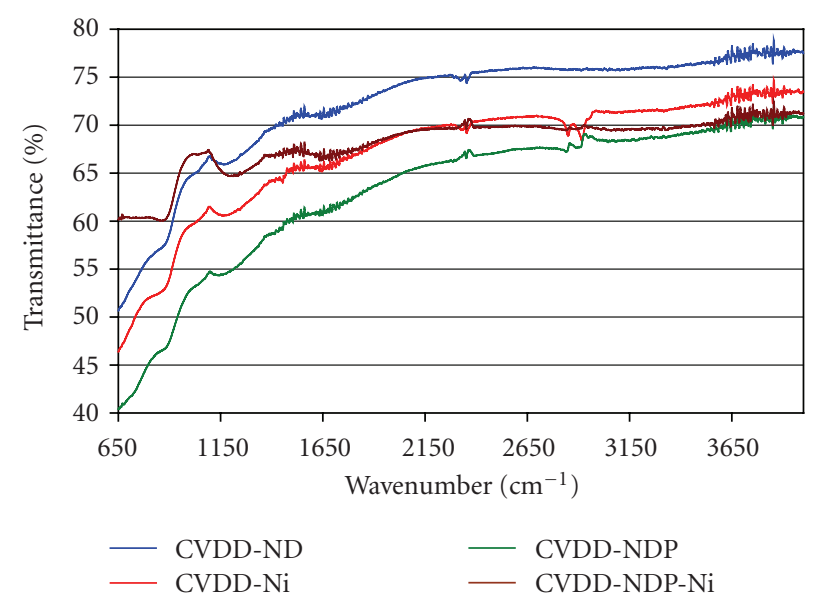

Figure 4: ATR/FT-IR spectra of diamond films.

$\left.1800 \mathrm{~cm}^{-1}\right)$ and carbon dioxide $\left(2350 \mathrm{~cm}^{-1}\right)$ is noticed in the spectra [19]. The $\mathrm{CH}$ and $\mathrm{CC}$ vibrational features of diamond films are located in the spectral ranges of $2600 \mathrm{~cm}^{-1}$ to $3200 \mathrm{~cm}^{-1}$ and $650 \mathrm{~cm}^{-1}$ to $1400 \mathrm{~cm}^{-1}$, respectively, as shown in Figure 5.

In Figure 5(a), the $\mathrm{sp}^{3}-\mathrm{CH}_{2}$ asymmetric and symmetric vibrations at $2918 \mathrm{~cm}^{-1}$ and $2850 \mathrm{~cm}^{-1}$ are distinctively observed in CVDD-Ni diamond films. Meanwhile, the additional $\mathrm{sp}^{3}-\mathrm{CH}$ vibration at $2894 \mathrm{~cm}^{-1}$ is found in CVDDNDP diamond films [20]. The incorporation of more hydrogen in CVDD-Ni diamond films may come from the catalytic dissociation of hydrogen which leads to hydrogenation of $t$ PA into methylene-like chains in the presence of nano- $\mathrm{Ni}$, as evidenced by Raman spectra in Figure 3. CVDD-NDP$\mathrm{Ni}$ diamond films indicate weak combinational features of CVDD-Ni and CVDD-NDP diamond films. However, it is almost featureless for CVDD-ND diamond film in the same $\mathrm{CH}$ vibrational region of $2600 \mathrm{~cm}^{-1}$ to $3200 \mathrm{~cm}^{-1}$. These spectroscopic observations might imply a better crystallinity of diamond crystals with less hydrogen included in CVDD-ND diamond films as evidenced by XRD in Figure 2. In Figure 5(b), the similar $\mathrm{C}-\mathrm{C}$ stretching features between $1000 \sim 1400 \mathrm{~cm}^{-1}$ and $\mathrm{C}-\mathrm{H}$ bending characteristics of disubstituted alkene-like structure around $870 \mathrm{~cm}^{-1}$ are observed in those diamond films [21].

3.5. X-Ray Photoelectron Spectroscopy of Diamond Films. In Figure 6, the XPS C 1s spectra of CVDD-NDP, CVDD$\mathrm{ND}, \mathrm{CVDD}-\mathrm{Ni}$, and CVDD-NDP-Ni diamond films (from bottom to top) are deconvoluted by Gaussian multiple peaks fitting into two main components with the binding energies (BE) of $281.2 \sim 281.3 \mathrm{eV}$ (for $\mathrm{sp}^{2}$ carbon bonding) and $281.6 \sim 284.0 \mathrm{eV}$ (for $\mathrm{sp}^{3}$ carbon bonding). Interestingly, the measured $\mathrm{C}$ 1s binding energies of our diamond films are relatively lower than the accepted $284.4 \mathrm{eV}$ for diamond [22], after a calibration by referring to $\mathrm{Cu} 2 \mathrm{p}_{3 / 2}$ peak in XPS spectra.

The highest binding energy of $\mathrm{sp}^{3}-\mathrm{C} 1 \mathrm{~s}$ peak and narrower distribution of CVDD-NDP diamond film suggest more $\mathrm{sp}^{3}-\mathrm{C}$ bonding present in the diamond film surface 


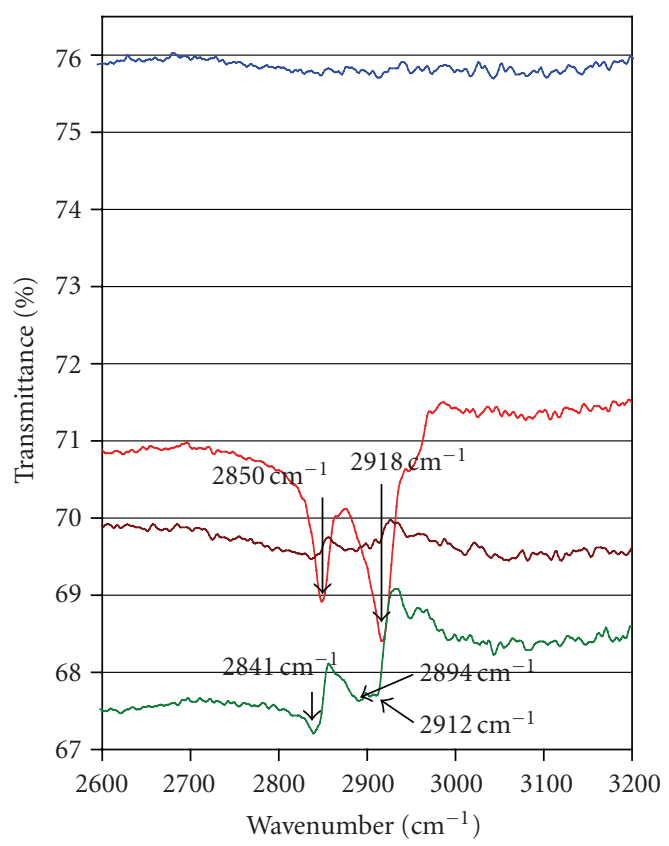

(a)

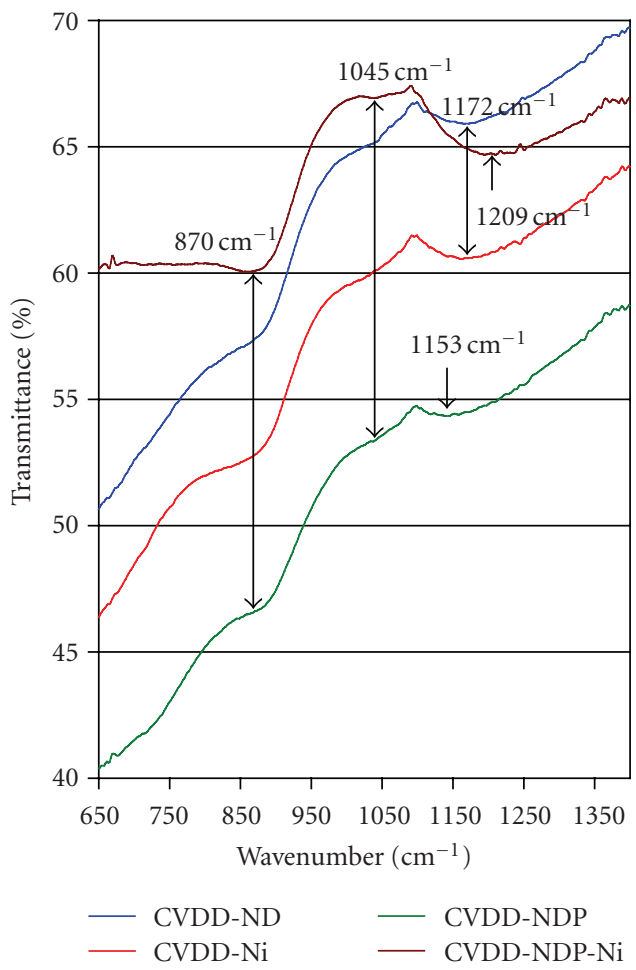

(b)

FIGURE 5: ATR/FT-IR spectra of diamond films in $\mathrm{CH}$ vibration region of $2600 \sim 3200 \mathrm{~cm}^{-1}$ (a) and CC vibration region of $600 \sim$ $1800 \mathrm{~cm}^{-1}$ (b).

than the others. On the other hand, the binding energies of $\mathrm{sp}^{2}-\mathrm{C} 1 \mathrm{~s}$ peaks are similar to all diamond films. However, the stronger $\mathrm{sp}^{2}-\mathrm{C} 1 \mathrm{~s}$ feature observed in both CVDD-Ni and CVDD-NDP-Ni diamond films could be due to the increasing formation and disorderness of amorphous carbon

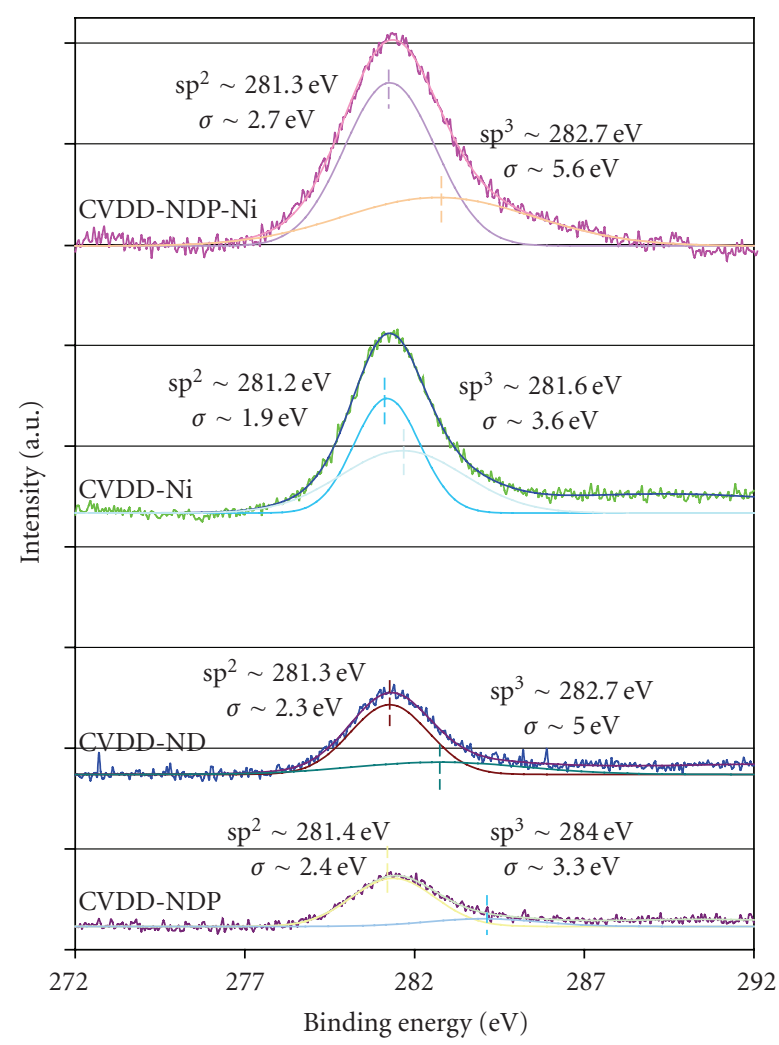

FIgURE 6: XPS C 1s spectra of diamond films with deconvoluted Gaussian fits of $\mathrm{sp}^{2}-$ and $\mathrm{sp}^{3}-\mathrm{C} 1 \mathrm{~s}$ peaks.

phase by the incorporation of nano-Ni into diamond films as illustrated in Raman and ATR/FT-IR spectra above.

3.6. TEM of Diamond Films. TEM pictures shown in Figure 7 are very informative for identifying the initial growing phases of diamond films as assisted by (a) ND, (b) nano$\mathrm{Ni}$, (c) NDP, and (d) nano-Ni/NDP mixture. For diamond crystals grown on diamond seed/powder, such as in Figures $7(\mathrm{a}), 7(\mathrm{c})$ and $7(\mathrm{~d}-2)$, the nanocrystalline diamond clusters were shown to be in overlapping hexagonal structures. However, in the right-lower corner of Figure 7(b), a short tube-like structure was observed on the surface of nano-Ni catalyst. Diamond crystals seem to be formed together with amorphous phase initially grown on nano-Ni catalysts. In Figure 7(d), nano-Ni catalysts clusters also were shown to be embedded in amorphous phase. The observed tube-like and amorphous layer forms on nano-Ni surface may lead us to speculate that those are the initial growing phases before a transformation from an amorphous phase to the crystalline diamond structures.

3.7. SEM and AFM Studies of Diamond Films. SEM pictures of diamond films grown on the pretreated $\mathrm{Si}$ substrates (a) CVDD-ND; (b) CVDD-Ni; (c) CVDD-NDP; (d) CVDDNDP-Ni) are shown in Figure 8. The pyramidal $\langle 111\rangle$ facets of diamond crystals are clearly observed. It is interesting to point out that the continuous polycrystalline diamond 

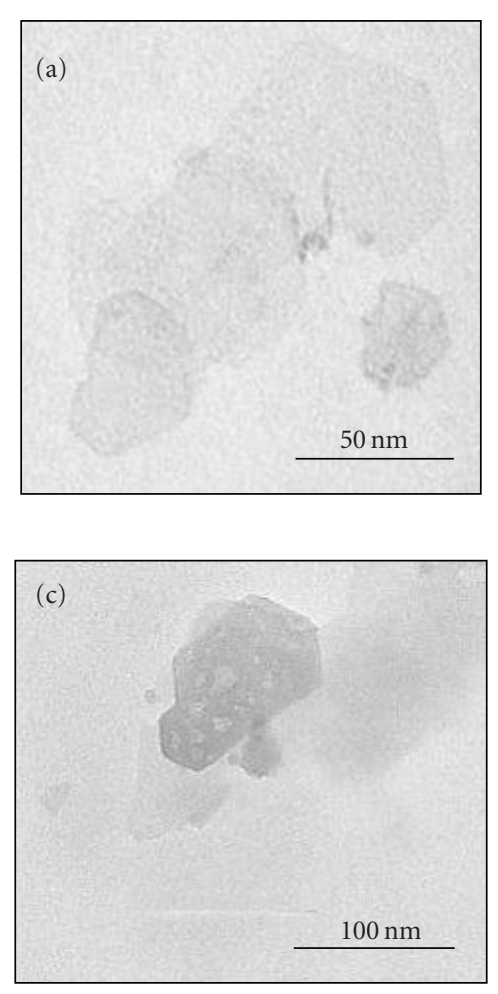

FIgure 7: TEM of diamond films: (a) CVDD-ND, (b)
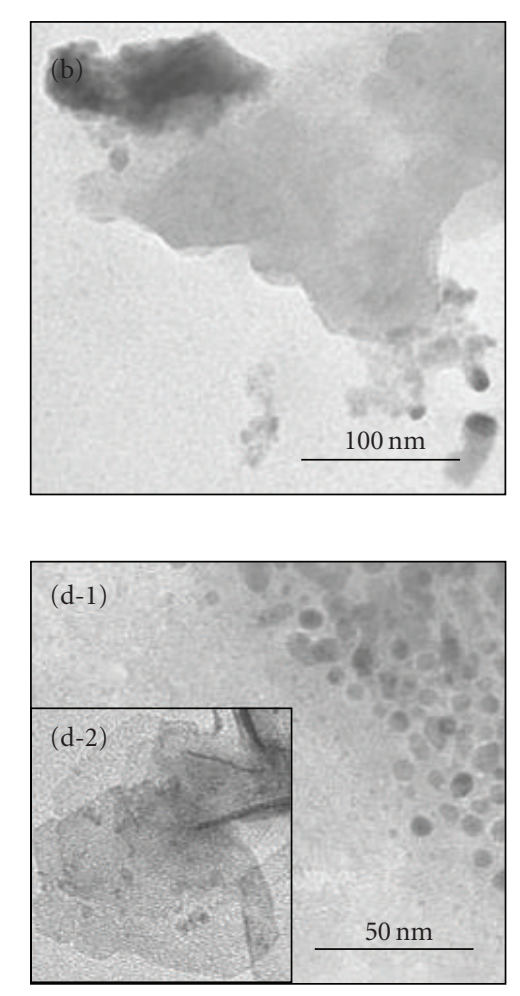

b) CVDD-Ni, (c) CVDD-NDP, and (d) CVDD-NDP-Ni. films were formed by the coalescence of the spherical cauliflower-like microcrystalline diamond particles about $20 \mu \mathrm{m}$ in size as shown in Figures $8(\mathrm{~b})$ and $8(\mathrm{c})$. Those coalescing boundaries are somehow clearly seen in the diamond films as shown in Figure 8(d). The formation of spherical cauliflower-like microcrystalline diamond particles may result from the isotropic nucleation and growth of diamond crystals on nanodiamond seeds and diamond powders. On the surface of nano-Ni catalysts, however, amorphous carbon phase may be formed initially at lower temperature of $700^{\circ} \mathrm{C}$ and followed by the nucleation of diamond crystals and then transformed to the spherical cauliflower-like microcrystalline diamond particles as shown in Figure 8(b). The continuous faceted diamond films grown on differently pretreated $\mathrm{Si}$ substrates are quite similar at first glance. The detailed examination shows that the diamond films grown on ND surface gave the better crystalline diamond particles as demonstrated by XRD analysis. The diamond films assisted by nano-Ni catalysts seem to have the diamond crystals of smaller size and less crystallinity than those seeded with ND and NDP as shown in Figure 8(b).

The smaller diamond crystals assisted by nano-Ni as compared to those grown directly on ND can also be evidenced by AFM morphology scans in Figure 9. The surface roughness (Rt) of CVDD-ND and CVDD-Ni diamond films was $1.552 \mu \mathrm{m}$ and $0.905 \mu \mathrm{m}$ as shown in Figures 9(a) and 9 (b), respectively. A $36 \%$ reduction in surface roughness for diamond film grown assisted by nano- $\mathrm{Ni}$ is quite surprising.
3.8. Effect of Nano-Ni on the Growth Mechanism of Diamond Films. When $\mathrm{CH}_{4} / \mathrm{H}_{2}$ gas mixtures were used for diamond film growth by HFCVD, the dominant mechanistic species above $\mathrm{H}$-terminated diamond growing surfaces were observed to be methyl radicals and acetylene [7]. The $\mathrm{CH}_{4}$ generates methyl radical and $\mathrm{H}_{2}$ provides the $\mathrm{H}$ source for hydrogen abstraction on diamond growing surfaces. The growth of diamond crystals proceeded through the successive addition of methyl radicals and followed by a C-C bridging of two $\mathrm{CH}_{3} \bullet$ bonded on adjacent carbon radical sites of diamond growing surface after hydrogen abstraction. Therefore, the concentration ratio of reactive species $\mathrm{H} / \mathrm{CH}_{3} \bullet$ plays several key roles in the growth of CVD diamond films. For example, $\mathrm{H}$ can be responsible for (1) activation of $\mathrm{H}$-terminated diamond growing surfaces by $\mathrm{H}$-abstraction, (2) etching or suppression of graphite and disordered carbons phases, (3) saturating dangling bonds on the diamond growing surfaces, and (4) incorporation into diamond films leading to the formation of amorphous hydrogenated carbon a-C:H or t-PA phases in diamond films [13-17].

The above mechanism is in agreement with of our experimental results of diamond films assisted by nanocatalysts and HFCVD using $\mathrm{CH}_{4} / \mathrm{H}_{2}$ gas mixtures. The compositions of diamond films show (1) crystalline diamond and amorphous (locally mixed $\mathrm{sp}^{3} / \mathrm{sp}^{2}-\mathrm{C}$ configuration) carbon phases and (2) $\mathrm{sp}^{2}$-bonded carbon clusters (short chain polyethylene such as t-PA in diamond films). Particularly, in our nano-Ni assisted growth of diamond films, the enhanced formation of 


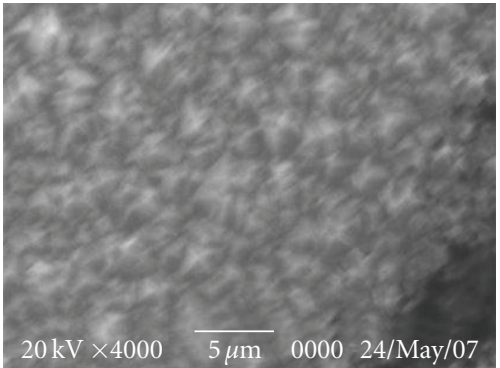

(a)

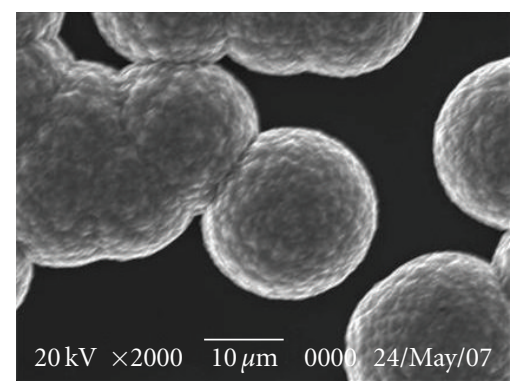

(c)

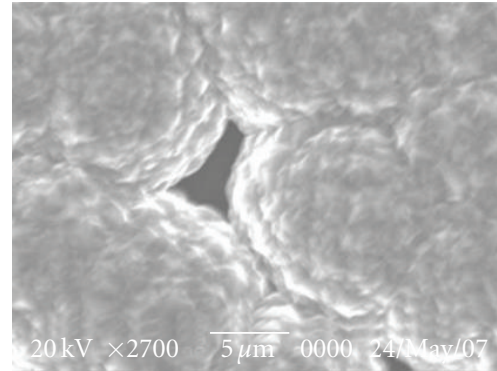

(b)

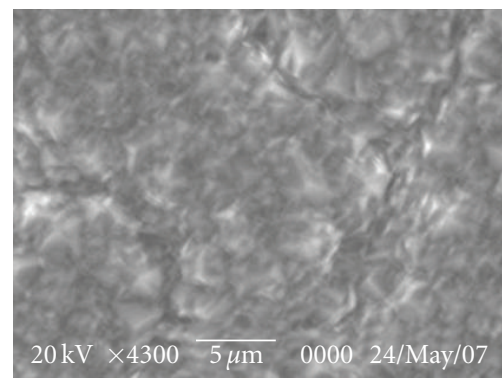

(d)

Figure 8: SEM of diamond films: (a) CVDD-ND, (b) CVDD-Ni, (c) CVDD-NDP and (d) CVDD-NDP-Ni.

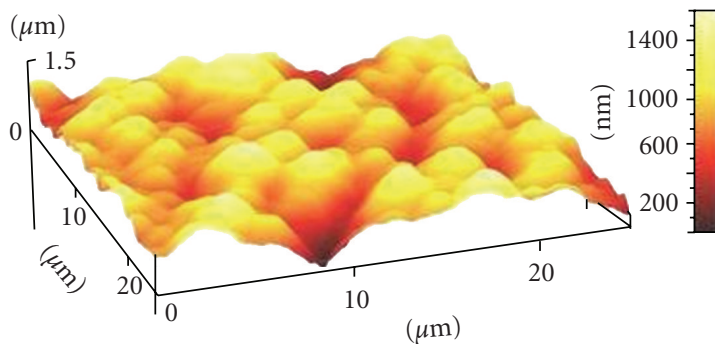

(a) Rt $1.4 \mu \mathrm{m}$

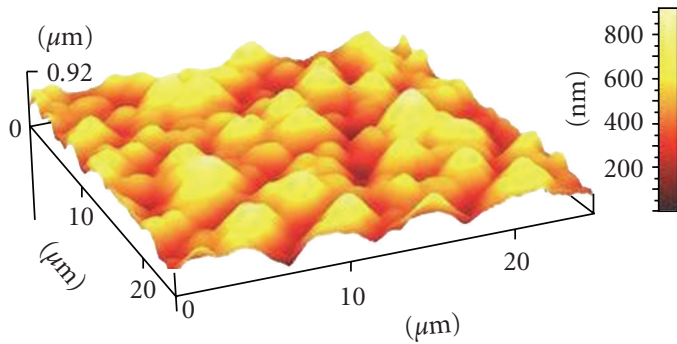

(b) Rt $0.9 \mu \mathrm{m}$

FIgURE 9: AFM morphologies of diamond films: (a) CVDD-ND and (b) CVDD-Ni.

$\mathrm{H}$ atoms by a catalytic dissociation of $\mathrm{H}_{2}$ on nano-Ni surface can subsequently activate the hydrogen abstraction reaction, saturate the dangling bonds on the diamond growing surfaces, and hydrogenate t-PA into methylene-like phases. This growth mechanism would lead to carbon deposition surrounding nano-Ni catalysts through the initial formation of glassy or amorphous carbons overlayers and followed by the nucleation of diamond crystals [3]. Meanwhile, the formation of the amorphous carbon layer on nano$\mathrm{Ni}$ catalyst surface may passivate the possible conversion of diamond to graphite in the presence of nano-Ni. From there on, new diamond nuclei grew on amorphous carbon layer, leading to the formation of spherical cauliflower-like microcrystalline diamond particles prior to coalescence into continuous diamond films.

\section{Conclusions}

We have demonstrated the growth of diamond films assisted by ND, nano-Ni, NDP, and nano-Ni/NDP mixture by
HFCVD using $\mathrm{CH}_{4} / \mathrm{H}_{2}$ gas mixtures. The growth of diamond films assisted by nanocatalysts has followed the $\mathrm{CH}_{3}$ • addition mechanism which leads to the observed spectral characteristics of diamond, amorphous carbon, and t-PA phases. The initial growth of diamond films seems to start with the $\langle 220\rangle$ (or $\langle 110\rangle$ ) facets which leads to the formation of diamond films of $\langle 111\rangle$ pyramidal faceted diamond crystals at the end as shown by XRD. The TEM and SEM results indicated that diamond crystals were grown directly on both large NDP and small ND seeds, but the latter displayed a better crystallinity of diamond films. The Raman spectral band at $2270 \mathrm{~cm}^{-1}$ has been verified to originate from the short chain t-PA formed in diamond films.

It was illustrated that nano-Ni nanocatalysts can promote the formation of amorphous carbon but suppress t-PA phases at the initial growth of diamond films. The subsequent nucleation and growth of diamond crystals on the amorphous carbon layer leads to generation of the spherical diamond particles and clusters prior to coalescence into continuous diamond films. Moreover, a 36\% reduction 
in surface roughness for diamond film assisted by nano-Ni catalyst is significant.

\section{Acknowledgments}

This work was supported by Kinik Company in Taiwan and Institute for Nano Science, Engineering and Technology of Northern Illinois University, USA. The authors would like to thank Dr. Laurence Lurio, Dr. Ralph Benbow, Dr. Michael Haji-Shiekh, Dr. Chong Zheng, and Dr. Heike Hofstetter for their technical assistance on DLS, XPS, AFM, XRD, and FTIR measurements.

\section{References}

[1] M. Yudasaka, R. Kikuchi, T. Matsui, Y. Ohki, and S. Yoshimura, "Specific conditions for Ni catalyzed carbon nanotube growth by chemical vapor deposition," Applied Physics Letters, vol. 67, no. 17, pp. 2477-2479, 1995.

[2] M. Mauger, V. T. Binh, A. Levesque, and D. Guillot, "Freestanding vertically aligned arrays of individual carbon nanotubes on metallic substrates for field emission cathodes," Applied Physics Letters, vol. 85, no. 2, pp. 305-307, 2004.

[3] D. N. Belton and S. J. Schmieg, "Loss of epitaxy during diamond film growth on ordered $\mathrm{Ni}(100)$," Journal of Applied Physics, vol. 66, no. 9, pp. 4223-4229, 1989.

[4] P. C. Yang, W. Zhu, and J. T. Glass, "Diamond nucleation on nickel substrates seededwith non-diamond carbon," Journal of Materials Research, vol. 9, no. 5, pp. 1063-1066, 1994.

[5] P. C. Yang, W. Zhu, and J. T. Glass, "Nucleation of oriented diamond films on nickel substrates," Journal of Materials Research, vol. 8, no. 8, pp. 1773-1776, 1993.

[6] E. Johansson, P. Skytt, J.-O. Carlsson, N. Wassdahl, and J. Nordgren, "Carbon growth in diamond deposition on nickel studied in situ by soft X-ray emission spectroscopy," Journal of Applied Physics, vol. 79, no. 9, pp. 7248-7255, 1996.

[7] W. Zhu, P. C. Yang, and J. T. Glass, "Oriented diamond films grown on nickel substrates," Applied Physics Letters, vol. 63, no. 12, pp. 1640-1642, 1993.

[8] Q. Yang, C. Xiao, W. Chen, and A. Hirose, "Selective growth of diamond and carbon nanostructures by hot filament chemical vapor deposition," Diamond and Related Materials, vol. 13, no. 3, pp. 433-437, 2004.

[9] Y. Chakk, R. Brener, and A. Hoffman, "Enhancement of diamond nucleation by ultrasonic substrate abrasion with a mixture of metal and diamond particles," Applied Physics Letters, vol. 66, no. 21, pp. 2819-2821, 1995.

[10] Y. Chakk, R. Brener, and A. Hoffman, "Mechanism of diamond formation on substrates abraded with a mixture of diamond and metal powders," Diamond and Related Materials, vol. 5, no. 3-5, pp. 286-291, 1996.

[11] J. Birrell, J. E. Gerbi, O. Auciello, J. M. Gibson, J. Johnson, and J. A. Carlisle, "Interpretation of the Raman spectra of ultrananocrystalline diamond," Diamond and Related Materials, vol. 14, no. 1, pp. 86-92, 2005.

[12] D. V. Musale, S. R. Sainkar, and S. T. Kshirsagar, "Raman, photoluminescence and morphological studies of Si- and Ndoped diamonds films grown on $\mathrm{Si}(100)$ substrate by hotfilament chemical vapor deposition technique," Diamond and Related Materials, vol. 11, no. 1, pp. 75-86, 2002.

[13] D. Roy, Z. H. Barber, and T. W. Clyne, "Ag nanoparticle induced surface enhanced Raman spectroscopy of chemical vapor deposition diamond thin films prepared by hot filament chemical vapor deposition," Journal of Applied Physics, vol. 91, no. 9, pp. 6085-6088, 2002.

[14] A. C. Ferrari and J. Robertson, "Origin of the $1150-\mathrm{cm}^{-1}$ Raman mode in nanocrystalline diamond," Physical Review B, vol. 63, no. 12, Article ID 121405, 4 pages, 2001.

[15] T. López-Ríos, É Sandré, S. Leclercq, and É Sauvain, "Polyacetylene in diamond films evidenced by surface enhanced Raman scattering," Physical Review Letters, vol. 76, no. 26, pp. 4935-4938, 1996.

[16] I. I. Vlasov, V. G. Ralchenko, E. Goovaerts, A. V. Saveliev, and M. V. Kanzyuba, "Bulk and surface-enhanced Raman spectroscopy of nitrogen-doped ultrananocrystalline diamond films," Physica Status Solidi A, vol. 203, no. 12, pp. 3028-3035, 2006.

[17] Sh. Michaelson, O. Ternyak, A. Hoffman, and Y. Lifshitz, "Hydrogen incorporation processes in nanodiamond films studied by isotopic induced modifications of Raman spectra," Applied Physics Letters, vol. 89, no. 13, Article ID 131918, 3 pages, 2006.

[18] L.-T. S. Lin, G. Popovici, M. A. Prelas, S. Khasawinah, and T. Sung, "Photoluminescence of free standing Hot-filament CVD diamond films, using He-Ne laser," Journal of Chemical Vapor Deposition, vol. 3, pp. 102-114, 1994.

[19] P. Thongnopkun and S. Ekgasit, "Attenuated total reflection Fourier transform infrared spectra of faceted diamonds," Analytica Chimica Acta, vol. 576, no. 1, pp. 130-135, 2006.

[20] P. John, D. K. Milne, I. C. Drummond, J. I. B. Wilson, M. J. Jubber, and J. A. Savage, "Attenuated total reflection infrared absorption in CVD diamond films," in Diamond Optics V, vol. 1759 of Proceedings of SPIE, pp. 209-217, San Diego, Calif, USA, 1992.

[21] D. L. Pavia, G. M. Lampman, and G. S. Kriz, Eds., Introduction to Spectroscopy, Saunders College Publishing, Philadelphia, PA, USA, 2nd edition, 1996.

[22] Y. Fan, A. G. Fitzgerald, P. John, C. E. Troupe, and J. I. B. Wilson, "X-ray photoelectron spectroscopy studies of CVD diamond films," Surface and Interface Analysis, vol. 34, no. 1, pp. 703-707, 2002. 

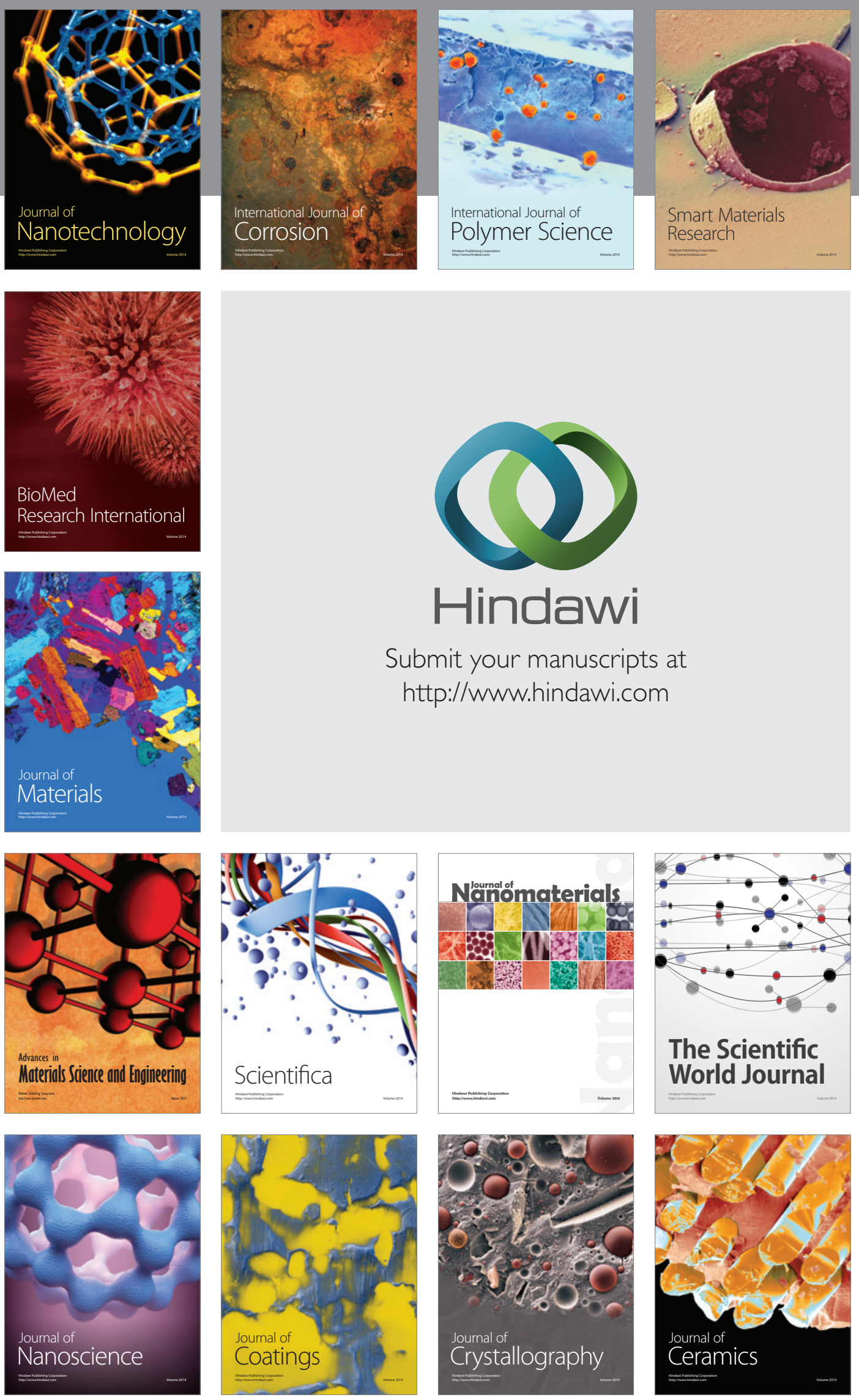

The Scientific World Journal

Submit your manuscripts at

http://www.hindawi.com

\section{World Journal}

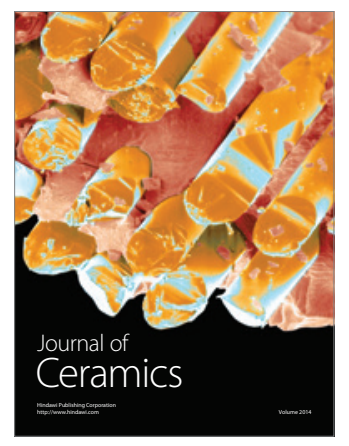

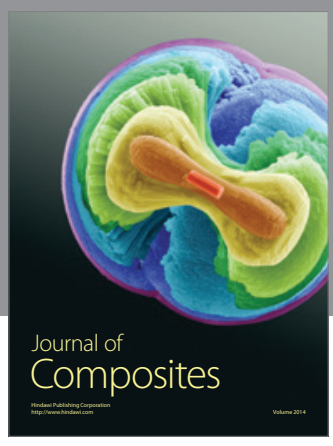
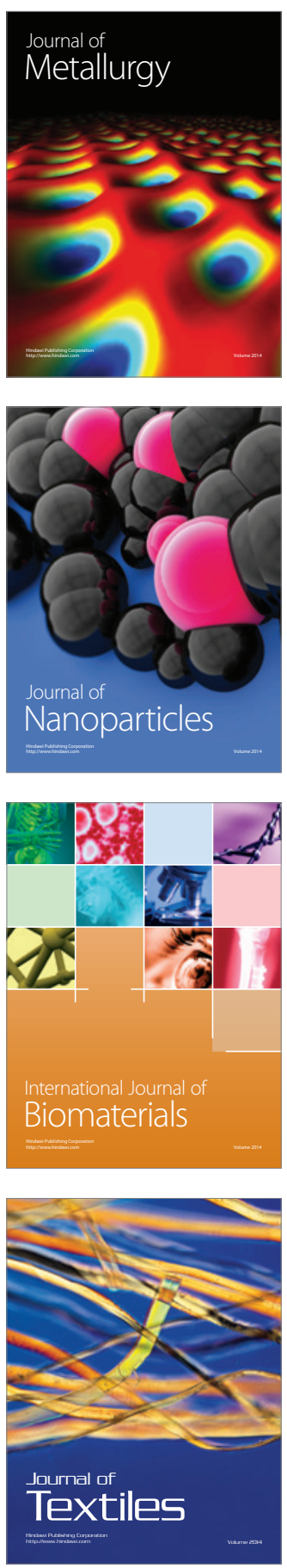